\title{
Look what I have felt: Unidentified haptic line drawings are identified after sketching
}

\author{
Maarten W.A. Wijntjes ${ }^{a, *}$, Thijs van Lienen ${ }^{a}$, Ilse M. Verstijnen ${ }^{b}$, Astrid M.L. Kappers ${ }^{a}$ \\ ${ }^{a}$ Utrecht University, Helmholtz Institute, Physics of Man, Princetonplein 5, Utrecht, Netherlands \\ ${ }^{\mathrm{b}}$ Utrecht University, Helmholtz Institute, Psychonomics, Princetonplein 5, Utrecht, Netherlands
}

Received 19 July 2007; received in revised form 26 January 2008; accepted 29 January 2008

Available online 2 April 2008

\begin{abstract}
The difficulty that observers experience when trying to identify a raised line drawing by touch is still largely unexplained. In this article, we show that observers who are unable to haptically identify a raised line drawing are suddenly able to do so after they have sketched on paper what they have in their mind. We conducted three experiments: first of all we show that this effect is robust; in the second experiment, we show that identification-after-sketching is caused by visual inspection of the sketch, and not caused by feedback in general; and in the third we show that sketches which were identified by the observers who produced them, were also identified by completely naive viewers. These experiments demonstrate that during raised line drawing identification the mental capacities required to interpret the stimulus seem to be inadequate: although enough pictorial information was present to produce a sketch which could even be identified by naive viewers, the stimulus could not be identified by haptic and mental processing alone. Furthermore, we investigated whether increasing the haptic perceptual field by using two hands instead of one hand had an influence on identification performance. We did indeed find that using two hands significantly increased identification. We use both results to discuss the underlying mechanisms of haptic raised line drawing identification.
\end{abstract}

(C) 2008 Elsevier B.V. All rights reserved.

PsycINFO classification: 2320

Keywords: Haptic line drawing perception; Imagery; Object identification; Externalisation

\section{Introduction}

"I bet you won't be able to identify an embossed line drawing by touch". Most people will take on the bet, but will lose it. At first sight the task seems easy, but when a blindfold is on and the fingers are exploring the raised lines, all self-confidence collapses. As noted by Hayward (in press), this can be demonstrated easily in a do-it-yourself experiment: sketch a picture with a pencil while pressing hard on the paper which is lying on soft backing; the back will be slightly embossed and perceptible by touch.

\footnotetext{
Corresponding author. Tel.: +3130253 7715; fax: +31302522664.

E-mail address: M.W.A.Wijntjes@phys.uu.nl (M.W.A. Wijntjes).

$U R L$ : www.phys.uu.nl/ wijnt102 (M.W.A. Wijntjes).
}

Another way to illustrate what makes haptic line drawing identification so difficult is to cut a small hole in an opaque sheet of paper and use that as an aperture. Superimposing the aperture sheet on a line drawing serialises the normally parallel visual input. Observers can move the aperture along the lines of the drawing and experience the same kind of difficulty as they would encounter when haptically exploring an embossed version.

Better controlled versions of these two do-it-yourself examples have been used in the past to investigate tactile picture identification. Raised line drawings are normally fabricated with a drawing kit or using swell paper. The drawing kit makes use of a pressure sensitive plastic sheet which becomes embossed when sketched on with a pen. Swell paper is made of heat-sensitive material which 
embosses the black, printed parts when heated. These two widely available tools have facilitated numerous studies on haptic identification of line drawings.

Compared to visual identification, haptic line drawing identification is characterised by low accuracy and long reaction times. To understand these characteristics, we will briefly review recent literature. Magee and Kennedy (1980) investigated the effect of the exploration mode on identification. In one condition, observers had to explore the pictures actively, in the other condition the observers were passively guided along the lines. The authors found that guided observers identified the raised line drawings more accurately than did the active exploration group. This was attributed to interference between movement control and object identification. If the observer does not have to continuously plan his movement in the local line direction sensed by the cutaneous receptors, then the observer can interpret the spatial layout better. The same study showed that observers can even identify a drawing when the fingertip is guided along a non-embossed line. Thus, the cutaneous information is predominantly used for movement control and not for identification. Along the same lines, Thompson, Chronicle, and Collins (2003) found that completely embossed drawings are better identified than raised line drawings. An embossed surface guides the finger more easily along the picture than only a line, thus relieving the observer partly from the exploration task. That an observer is actually performing a dual task of exploration and identification is evidently an important factor responsible for the low identification latencies. Another important contributor to the difficulty of haptic line drawing perception is the constraint that the fingers can only extract the spatial information in a serial manner. Loomis, Klatzky, and Lederman (1991) experimentally 'serialised' vision by constraining the field of view to match the size of a fingertip. They found that identification became just as difficult for vision as it was for touching with a single finger. Together, these findings illustrate two important restricting factors of raised line drawing identification: exploration interferes with identification and (haptic) serial information acquisition is slower than (visual) parallel processing. From these findings, we can understand at least one characteristic of raised line drawing identification: the slow reaction times. However, these findings do not explain why observers sometimes only reach $25 \%$ accuracy (e.g., Heller, Calcaterra, Burson, \& Tyler, 1996) despite unlimited exploration time. Observers seem to lack some perceptual or cognitive capabilities to identify a line drawing by touch. Intuitively this may not be surprising, since the purpose of line drawings is to communicate ideas via the visual sense. On the contrary, it may seem very surprising that line drawings can be identified by touch at all.

Two theories have been proposed to explain haptic line drawing identification. The origins of these two ideas can be found in studies in which the role of visual experience is investigated. In these studies, three kinds of observers are compared: congenitally blind, who clearly do not have any visual experience but might have a better developed haptic sensitivity than sighted observers; late blind, who do have visual experience and also might have haptic superiority; and lastly blindfolded sighted, who have visual experience but may have a less developed haptic sensitivity. Two studies addressing the issue of visual experience contradict each other: Heller (1989) found that the performance of congenitally blind observers is similar to that of sighted observers, whereas Lederman, Klatzky, Chataway, and Summers (1990) found that congenitally blind observers performed more poorly than sighted observers. From this contradiction two theories emerged.

The first theory was outlined by Kennedy (1993) and was commented on by D'Angiulli, Kennedy, and Heller (1998) and Kennedy and Bai (2002). These authors argue that no visual experience is needed to interpret the various pictorial meanings of line configurations like edges. Also, concepts such as vantage points, outlines and perspective apply to the haptic perception of raised line drawings, irrespective of the observers' visual experience. It is proposed that identification of line drawings is an amodal process, i.e., both the visual and haptic modality can be used to interpret line configurations. The difference in identification performance is more a matter of experience than a fundamental difference between modalities. Further evidence for this theory was found in case studies of congenitally blind observers (e.g., Kennedy \& Juricevic, 2006) and more general studies on haptic perception of perspective (Heller, 2002).

Lederman et al. (1990) proposed a different theory to explain raised line drawing identification. In their study, they found evidence that identification is mediated by visual imagery, i.e., the observer translates the haptic spatial layout into a visual image which is identified using visual experience. This hypothesis explains their finding that congenitally blind observers (who do not have visual experience) perform more poorly than late blind and sighted observers (who both have visual experience). According to this theory the difference in identification performance has a modality-specific cause. The haptic modality is unable to identify line drawings and observers need to translate the stimulus into a visual mental image in order to identify it.

Since both theories are supported by experimental evidence, the question of what mediates haptic line drawing identification is still unsolved. In the present study, we will test a hypothesis that could sharpen and even resolve this debate. Ikeda and Uchikawa (1978) reported a case in which an observer, who initially did not identify a raised line drawing by touch alone, could suddenly do so after taking off his blindfold and sketching the image on paper. This was only a single observation but the implications of such a mechanism would help us to gain a better understanding of haptic line drawing identification. If this finding can be generalised, it would contradict the amodal hypothesis of Kennedy (1993) since it would prove that feedback of tactual information into the visual system enhances identification. Furthermore, it would mean that the imagery process (Lederman et al., 1990) has some lim- 
itations in comparison to direct visual input. In the experiments presented in this paper, we investigated the identification-after-sketching effect by asking observers to explore raised line drawings for a fixed time interval. After exploration, first of all they had to name the object (i.e., identify the basic-category to which the object belongs), secondly they were allowed to sketch the object, and thirdly they had to name the object again. If the observation made by Ikeda and Uchikawa (1978) can be generalised, we should find a substantial increase in identification after sketching. In the three experiments we investigated the identificationafter-sketching effect in detail. In the first experiment, we investigated whether this effect is robust and in the subsequent two experiments we tried to disentangle which component is crucial for the effect.

As noted above, various studies have investigated how we retrieve pictorial information from a raised line stimulus. Wijntjes, Lienen, Verstijnen, and Kappers (in press) found that large raised line drawings are better identified than small raised line drawings. It has also been found that using five fingers instead of one finger improved identification (Klatzky, Loomis, Lederman, Wake, \& Fujita, 1993). In contradiction to this latter finding, Loomis et al. (1991) found that the use of two adjacent fingers did not increase identification performance with respect to a single finger. Klatzky et al. (1993) also investigated the influence of manual restriction on real 3D object identification. While material properties were blocked by the use of a glove, the object geometry could be explored under three levels of restriction: with the whole hand, with only the five fingertips and with only one fingertip. They found that identification became increasingly difficult when the effective perceptual field was decreased. This suggests that for 3D object exploration identification is better when the size of the perceptual field is larger. As mentioned, raised line drawing identification increased using five fingers, but not using two fingers. A possible explanation for this contradiction is that the increment in perceptual field size was too small to be measured for the two-finger condition. Our first experiment aims to resolve this issue by comparing one- and two-hand exploration. If two-handed exploration yields better identification, then it is likely that the same perceptual field size principle holds for $2 \mathrm{D}$ and $3 \mathrm{D}$ objects.

\section{Experiment 1}

In the first experiment we investigated whether identification-after-sketching is a robust phenomenon. In addition, we investigated the influence of hand use on identification.

\subsection{Method}

\subsubsection{Participants}

A total of 20 sighted observers (10 men and 10 women) participated in this study, five of whom were financially rewarded. All participants were naive with respect to the purpose of the experiment and had no knowledge of the raised line stimuli.

\subsubsection{Stimuli and materials}

Twenty raised line drawings were used. The stimuli represented a wide range of objects, as can be seen in Fig 1. Some pictures were inspired by previous studies. The raised lines were produced with Zytex Swellpaper (http://www.zychem-ltd.co.uk/). The lines were $1 \mathrm{~mm}$ wide, approximately $0.5 \mathrm{~mm}$ high and the sizes of the pictures were scaled to fit onto a sheet of A4 $(21 \times 29.7 \mathrm{~cm})$ paper with $2 \mathrm{~cm}$ margins. This size is relatively large with respect to other studies but we decided to use large stimuli because our previous research (Wijntjes et al., in press) indicated that size positively influences identification.

\subsection{Procedure}

Participants received written instructions explaining the procedure. They were blindfolded when exploring the drawings haptically. The total exploration time was $45 \mathrm{~s}$. At $30 \mathrm{~s}$ a signal was given that $15 \mathrm{~s}$ remained. At the end of the fixed exploration time of $45 \mathrm{~s}$, the participants had to identify the object that was represented. They were told explicitly that even if they had only a vague idea they were supposed to communicate it. No feedback was given about the correctness of their responses. After their response, participants had to reproduce the picture by sketching with a pencil on a blank sheet of paper. The participants always had to draw, irrespective of the correctness of the first response. Since possible identifications could be caused by the extra thinking time during the sketching phase, we introduced a control condition in which observers kept their blindfold on. In that way, they were performing the same motor act in approximately the same amount of time, but were not able to perceive their sketch visually. Participants could take as long as they needed to finish their drawing. After drawing, they had to name the object again. If the drawing was done blindfolded, the blindfold remained on during the naming procedure. They were told that they could repeat their initial response (i.e., the response they gave before drawing) or give a new response.

Concerning our first research question, the only interesting trials are those which initially were named incorrectly. We distributed these non-identified trials equally among the conditions of sketching with and without blindfold. To do so we assigned the sketching condition depending on the responses. We designed a protocol which allowed the sketching condition to alternate independently for the correct and the incorrect trials. An example of a hypothetical series is presented in Table 1. As can be seen, the sketching condition switches between with and without blindfold independently for the correct and incorrect responses.

Furthermore, hand use was controlled during the experiment. Half of the participants had to use only one hand during the first ten trials and two hands during the last 

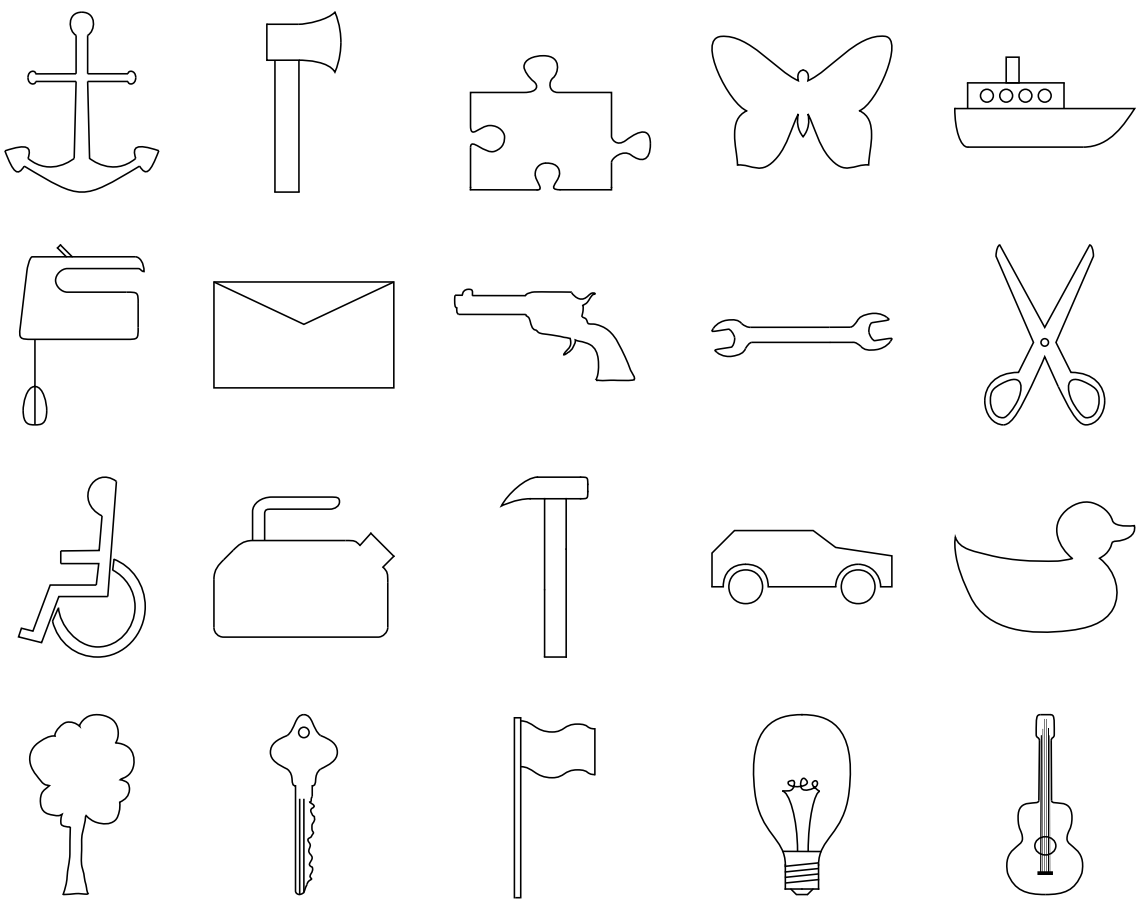

Fig. 1. From right to left and from top to bottom: (1) anchor; (2) axe; (3) piece of jigsaw puzzle; (4) butterfly; (5) boat; (6) kitchen mixer; (7) envelope; (8) revolver; (9) open-end wrench; (10) scissors; (11) handicapped sign; (12) kettle; (13) hammer; (14) car; (15) duck; (16) tree; (17) key; (18) flag; (19) light bulb; and (20) guitar. During the experiment the stimuli were presented in this order.

Table 1

Hypothetical series of the condition assignment during experiment 1

\begin{tabular}{|c|c|c|c|c|c|c|c|c|c|c|c|c|c|c|}
\hline Picture & & 1 & 2 & 3 & 4 & 5 & 6 & 7 & 8 & 9 & 10 & 11 & 12 & etc. \\
\hline \multirow[t]{2}{*}{ Correct } & Blindfolded & $\mathrm{x}$ & & & $\mathrm{x}$ & & & $\mathrm{x}$ & & & & & & \\
\hline & Not blindfolded & & $\mathrm{x}$ & & & & $\mathrm{x}$ & & & & & $\mathrm{x}$ & & \\
\hline Incorrect & Blindfolded & & & $\mathrm{x}$ & & & & & $\mathrm{x}$ & & $\mathrm{x}$ & & & \\
\hline
\end{tabular}

In this case the starting conditions were both blindfolded. As can be seen, the first two drawings were correctly identified and the third trial was incorrect, etc.

ten trials. This order was reversed for the other half of the participants. When one hand was used, participants were instructed to use the preferred hand. Participants were free to choose how many fingers to use during exploration. In general, the number of fingers used for exploration did not seem to depend on whether one or two hands were used. Thus, the perceptual field increased with the number of hands used for exploration.

\subsubsection{Analysis}

The total number of trials for 20 participants and 20 drawings amounted to 400 . We fixed the exploration time at $45 \mathrm{~s}$ because we estimated that approximately half of the stimuli would be correctly identified at first response (before drawing). This estimate was based on the median response latency of $37 \mathrm{~s}$ found in previous research (Wijntjes et al., in press). Since the median identification latency is by definition based only on correct responses we adjusted the time limit upwards. We analysed the effect of sketching with or without blindfold by determining the $z$-score on the two independent proportions for the two sketching conditions. Furthermore, we tested the homogeneity of the effect by analysing whether the identification-after-externalisation occurred both for the various participants and for multiple stimuli. This was to ensure that if there was an effect, it was not due to either specific participants or specific stimuli.

We analysed the effect of hand use independently of the sketching conditions. Only the first answer was analysed with respect to the hand use analysis. The hand conditions can only affect the sketching effect, and not vice versa. The effect of hand use was analysed using a paired $t$-test on the average accuracy scores per participant. Since our previous research suggested that bimanual exploration may enhance identification we used a one-tailed $p$-value.

\subsection{Results}

A diagram illustrating the overall results is presented in Fig. 2. Only the responses which were initially incorrect are 


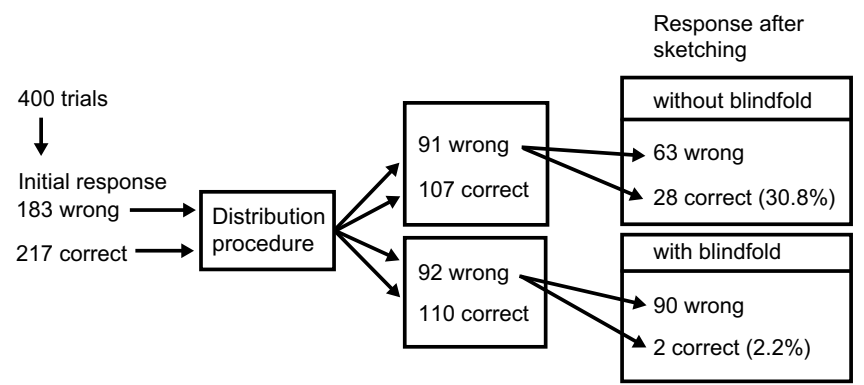

Fig. 2. Diagram illustrating the results of experiment 1 . The total number of trials was 400 (20 participants and 20 stimuli). The initially correct and incorrect trials were distributed equally among the sketching conditions as described by Table 1 . Almost one-third of the 91 initially not identified trials were identified after sketching without blindfold.

of interest for our research question. When participants could see while they were sketching, 28 'discoveries' occurred, whereas only 2 occurred when vision was blocked by the blindfold. This means identification occurred in $30.8 \%$ of the trials with vision, and in $2.2 \%$ of the trials without vision. This difference between the blindfold conditions was highly significant as revealed by a $z$-score for two independent proportions $(z=5.6$, $p<0.0001$ ). The effect was homogeneously spread over the participants and pictures: 16 participants showed at least one 'discovery' and 14 pictures were identified by at least one participant after drawing with visual feedback. Of the 217 initially correct identified stimuli, none was given a new (incorrect) name after the drawing procedure; in other words, no transitions from correct to incorrect occurred after the drawing phase.

The average identification accuracy for unimanual and bimanual exploration was $49.5 \%$ and $59.4 \%$, respectively. We conducted a paired $t$ test on the mean identification accuracy of the participants. This showed that identification was significantly better for bimanual exploration then for unimanual exploration $\left(t_{19}=2.703, p<0.01\right.$, onetailed). Furthermore, of the 28 visual 'discoveries', 17 were explored unimanually and 11 bimanually. We performed an independent proportion test which showed that this difference was not significant $(z=0.89, p=0.38)$.

\subsection{Discussion}

We have shown that identification-after-sketching is a robustly measurable phenomenon. Almost one-third of the raised line drawings which were not identified initially were correctly identified when they were sketched without blindfold, whereas hardly any were identified when vision was blocked by the blindfold. Furthermore, we found that bimanual exploration leads to better identification than does unimanual exploration. This last finding will be commented on further in the general discussion.

The identification scores are in line with our previous research. We limited the exploration time to $45 \mathrm{~s}$ which was the (adjusted) median of the reaction latencies reported by Wijntjes et al. (in press). Thus, we estimated that the identification accuracy would lie around $50 \%$. The average identification accuracy we found in experiment 1 was indeed $54.5 \%$. The accuracy rates in the present study and in Wijntjes et al. (in press) are higher than the rates normally found in the literature, which lie between $10 \%$ and $45 \%$. As was noted by Wijntjes et al. (in press), the size of the line drawings could be partly responsible for this. The size of the stimuli used in the present study was between 17 and $25.7 \mathrm{~cm}$ (depending on their aspect ratio), whereas other studies often used picture sizes between 8 and $17 \mathrm{~cm}$. An exception is the study by Kennedy and Bai (2002) who used pictures sizes ranging from 15 to $22 \mathrm{~cm}$ which resulted in a high identification rate, namely $61 \%$. The high accuracy in experiment 1 may also be due to the fact that our picture set differed from the set used in previous studies. Some researchers use the standardised set from Snodgrass and Vanderwart (1980), which is normally used in visual line drawing identification and others use their own set, for instance, Kennedy and Bai (2002) and Heller et al. (1996). In our previous study we used 12 pictures, 11 of which were also used in the present study. Since the present accuracy data are consistent with our previous study, it is likely that each set contains a certain inherent difficulty. Our particular set could thus be partly responsible for the high identification rates.

Our results clearly show that the observation made by Ikeda and Uchikawa (1978) is a robust phenomenon: sketching an unidentified pictorial stimulus can result in identification. To ensure that this effect was not caused by the extra time associated with the sketching condition we used a control condition in which the blindfold remained on during sketching. Thus, we can explain our finding by suggesting that visual feedback during sketching caused identification. However, we cannot actually distinguish whether it is feedback in general or specifically visual feedback that caused identification-after-sketching. We designed a second experiment to further investigate this distinction.

\section{Experiment 2}

In this experiment, we wanted to investigate whether haptic feedback after sketching would also lead to identification-after-sketching as visual feedback had shown in experiment 1 . In order to do this, we used a raised line drawing kit as sketching equipment. The drawing kit allows immediate haptic feedback since the pencil strokes cause clearly tangible relief. This drawing kit is often referred to as the 'Swedish drawing kit'.

\subsection{Method}

\subsubsection{Participants}

Twenty observers participated in this experiment. They were naive with respect to the purpose of the experiment and had no experience with the raised line stimuli. All were paid for their participation. 


\subsubsection{Stimuli and material}

We used the same stimuli as in experiment 1 . We used the raised line drawing kit as sketching medium. The essential ingredient of the raised line drawing kit is the plastic sheet which deforms when sketched on. The deformations establish thin but clearly tangible lines. The sheets were attached firmly so they could not move when the participants were sketching.

\subsubsection{Procedure}

Participants received written instructions explaining the procedure. Before the experiment started, the raised line drawing kit was shown to the participants and they were asked to sketch some lines to familiarise themselves with the kit. The participants were told that they were free to explore the stimuli in the way they wanted. The sketching always took place with the blindfold on. Otherwise, the procedure was similar to that in experiment 1 . Before the experiment started, one test trial (a raised line drawing of a lamp) was given in order to familiarise the participants with the procedure. During this trial the sketching procedure was also practised. As in the first experiment, identification was measured after haptic exploration and after the sketching procedure. The observers were told explicitly that after they had finished sketching the stimulus, they should touch their own sketch again before giving their final answer.

\subsection{Results}

In total 175 of the 400 trials were initially identified correctly, i.e., an average accuracy of $43.8 \%$. Since we found an average identification accuracy which was substantially lower than that found in experiment 1 , we performed an unpaired $t$-test on the mean accuracy scores of the participants from experiments 1 and 2. Since the hand use of participants in experiment 2 was not constrained we used the average accuracy scores for unimanual and bimanual exploration from experiment 1 for comparison. The $t$-test did not reveal a significant difference between the identification accuracy of the two experiments $\left(t_{38}=1.717\right.$, $p=0.094)$.

Of the 225 unidentified trials, $10(4.4 \%)$ were identified after sketching with the raised line drawing kit. This occurred for 8 different participants and for 9 different stimuli. The identification rate of experiment 2 did not significantly differ from the $2.2 \%$ found for the drawing with blindfold condition in experiment 1 as was revealed by a $z$ score for two independent proportions $(z=1.1, p=0.27)$. Compared to the visual feedback condition of experiment 1 there was a significant difference between the two proportions $(z=5.2, p<0.0001)$.

\subsection{Discussion}

The result of experiment 2 shows that using a raised line drawing kit does not improve the identification of unseen sketches. This finding rules out the possibility that identification-after-sketching is caused by a modality-independent feedback mechanism. Instead, identification-after-sketching seems to be a phenomenon specifically occurring when visual feedback of the sketch is provided.

In our last experiment, we wanted to investigate this visual feedback mechanism in more detail. Identification by an observer of his or her sketch in experiment 1 could be due to several factors. Firstly, the observer had haptically perceived the original picture; secondly, the observer could see the moving, sketching hand; thirdly, the observer is seeing the emerging sketch; and lastly the observer saw the final result. In order to partly disentangle these factors we conducted a third experiment.

\section{Experiment 3}

To understand why sketches led to identification we wanted to know more about how the sketches were used by the participants. Did their haptic knowledge (i.e., the experience of having touched the stimulus) contribute to the identification? Was it important to see the sketching hand or was merely seeing the resulting sketch sufficient for identification? We tried to answer this question by measuring how recognisable the sketches were to naive viewers. If naive viewers are able to identify a sketch, then haptic knowledge and seeing the sketching hand are likely to be of minor importance.

In the third experiment we only used the sketches which were identified when sketched without blindfold from experiment 1 . From experiment 1, video recordings were available for half of the observers who showed identification-after-sketching. We watched the recordings to check at what moment during sketching the identification occurred. This is relevant because it may influence the quality of the sketch: if identification occurs during sketching it is possible that the observer uses this knowledge to improve the sketch. The video recordings revealed that most of the time identification during experiment 1 occurred after sketching was finished (12 out of 14). This means that subjects generally completed their sketching before identification.

\subsection{Method}

\subsubsection{Participants}

Twenty-four observers who were naive with respect to the purpose of the experiment and had seen neither the stimuli nor the sketches participated in this experiment.

\subsubsection{Stimuli and materials}

The results from experiment 1 showed that only the raised line drawing of the duck resulted in four discoveries. We randomly left out one of these 4 duck sketches and from the resulting 27 sketches we constructed three sets in which no drawing was represented more than once. 


\subsubsection{Procedure}

Each set of nine sketches was shown to 8 participants. Participants were instructed to try to identify the sketch. They reported this by writing down their answers.

\subsection{Results and discussion}

The average identification scores are presented in Fig. 3. As can be seen, the sketches are identified more often than not. Only 3 sketches were identified by less than $50 \%$ of the observers and all of the sketches were identified at least once. On average $76 \%$ of the sketches were identified by naive viewers. These results suggest that in most cases haptic knowledge or seeing the sketching hand is not necessary for identification.

\section{General discussion}

Our experiments have shown that identification-aftersketching is a robust phenomenon and occurs specifically when a finished sketch is being inspected visually. Of the raised line drawings which were initially not identified, $30.8 \%$ became identifiable after the spatial information was externalised by means of sketching. We have also shown that sketches of unidentified stimuli are identified only by vision, and not by touch. Furthermore, the sketches were recognisable for naive viewers, suggesting that the sketch does not serve as a supplement to the identification task, but serves rather as an independent stimulus.

As outlined in the introduction, two theories compete for the explanation of raised line drawing identification, namely the amodal theory of Kennedy (1993) and the modality-specific theory of Lederman et al. (1990). At first sight, our experiments show that raised line drawing identification is a modality-specific problem, since identification increases when the stimulus perceived by the haptic modality is fed back through the visual modality. Thus, our findings favour the theory of Lederman et al. (1990). Since this

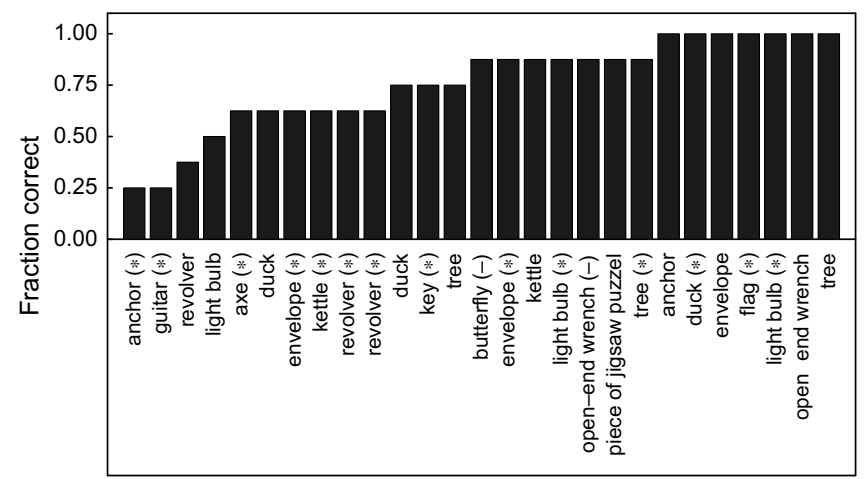

Fig. 3. Average identification scores for 27 sketches which led to identification in experiment 1 . The scores are ordered and the names of the original drawings are written on the $x$-axes. With regard to the sketches from which video recordings were available we report whether identification occurred during the sketch $(-)$ or after $(*)$. idea is based on the assumption that a mental image can be identified (i.e., visualisation can lead to identification), we need to understand the relation between imagery and perception. This relation is central to the so-called imagery debate about the nature of mental representations. Interestingly, researchers involved in this debate found identification-after-sketching effects similar to the ones we found.

The ongoing imagery debate focuses on the question of whether the mental representations used in an imagery task are depictive or descriptive. Kosslyn (1994) states that the representations are depictive and he takes the view that perception of a mental image is similar to perception of the real world. On the other hand, (Pylyshyn, 2003) states that imagery is purely descriptive; the vivid visual experience humans have when visualising an object or scene is merely an illusion. To resolve this debate, researchers performed experiments in which observers were asked to perceive mental images. One way of doing this is to show observers an ambiguous figure for a brief moment. If only one of the two referents is identified, the task is to identify the other referent using imagery. Chambers and Reisberg (1985) presented the rabbit/duck ambiguous figure for $5 \mathrm{~s}$. Whereas none of the participants could reinterpret the figure during imagery, all were able to identify the other referent after sketching. Since the imagery task did not cause identification but sketching did, the results were explained as an argument against the depictive hypothesis. A different paradigm was used by Finke, Pinker, and Farah (1989) who investigated the identification of mentally constructed images. Observers were given verbal instructions on how to manipulate and synthesise alpha-numerical symbols. For example: 'Imagine a capital letter 'D'. Rotate the figure 90 degrees counter-clockwise. Now attach a capital letter ' $J$ ' at the bottom." This instruction should lead to the identification of an umbrella. They found that observers could identify most mental constructs; this evidently was used as an argument counter to the descriptive hypothesis. However, Finke et al. (1989) also measured the effect of externalisation when identification failed. In their second experiment they found that $83 \%$ of the unidentified stimuli were identified after sketching.

Although not resolving the imagery debate, these studies showed that externalising a mental image can lead to identification. Together with our results these findings suggest that the externalisation effect is independent of the origin of the mental image: Externalisation increases identification rates both for visually (Chambers \& Reisberg, 1985), verbally (Finke et al., 1989) or haptically (our study) perceived pictures. It should be noted that externalisation is also often used in everyday life. Multiplying two numbers consisting of more than two digits makes one reach for a paper and pencil. Physics and mathematics students need to write out the symbolic derivations in order to understand complicated equations. More formally, Verstijnen, Leeuwen, Goldschmidt, Hamel, and Hennessey (1998) showed that externalisation is used when observers have a need to restructure a mental image. 
Externalisation seems to be a process which can be used to restructure uninterpretable input. In the case of line drawings, it restructures the serial input into parallel input. Looking at the sketch essentially recruits the large perceptual field of vision. A similar relation between the perceptual field size and identification was found in Experiment 1. There, we showed that two-handed exploration enhances identification with respect to one-handed exploration. This finding complements the finding of Klatzky et al. (1993) who found that five-finger exploration outperforms single finger exploration. On the assumption that the difference between one and two adjacent fingers (Loomis et al., 1991) was too small to be measurable, one is led to conclude that increasing the perceptual field positively influences perception. A functional equivalence holds for vision: increasing the aperture size increases identification rates. It can thus be hypothesised that the difference between visual and haptic line drawing perception is only a matter of different perceptual field sizes and is not a result of the visual system having privileged access to line drawing identification. It is interesting to note that blindfolded observers have a natural tendency to increase their perceptual field by using multiple fingers and two hands (Wijntjes et al., in press).

Why do we (sometimes) need to restructure a serially acquired line drawing? It could well be that the way line drawings are explored gives rise to a representation which cannot be matched to an internal representation and thus cannot be identified. The representation which is built up when the line drawing is being explored is of such a serial nature that it does not suit the identification system which uses parallel or simultaneous representations. Rephrasing, one could say that haptic line drawing exploration results in a one-dimensional description in which only left or right turns are coded, whereas the internal representations that are used for object identification are two-dimensional. One-dimensional descriptions lack configural regularities such as parallelism and symmetry which may play an important role in object identification as suggested by Panis, De Winter, Vandekerckhove, and Wagemans (2008). As one reviewer noted, the assumption that haptically perceived pictures are encoded one-dimensionally could be tested by investigating the video recordings. If exploratory movements could predict the sketching movements, this would be the evidence that observers use a one-dimensional description. This is a very interesting idea, but studying our video recordings made it clear that these could not be used for this purpose. As was noted by Wijntjes et al. (in press), observers usually explore using multiple fingers, even when using one hand only. This was also evident from the video recordings of the current experiment: since multiple fingers were exploring the drawing, we could not relate these movements to the production of the sketch. However, there is evidence from the literature that typical errors are made during the reproduction of haptically explored two-dimensional shapes. Becker (1935) investigated haptic perception of two- dimensional shapes which were composed of simple closed shapes such as circles, squares and triangles. Interesting configurations were the ones where two shapes partly overlapped. The first observation Becker made was that for two overlapping circles a large part of the observers did not detect the two circles but perceived either three non-overlapping closed shapes or they grouped the outer figure and the inner figure. He could not relate the exploration movements to the reproduction movements but he did observe that the way the stimulus is explored is at least partially responsible for the errors. In another experiment Becker (1935) showed that when vision is confined by an aperture, similar reproduction errors occur, although less frequently than with touch. Although the experiments were performed on children aged 9 to 11 years, informal observations from our lab confirm that for the two overlapping circles the typical errors were also made by adult observers: 10 out of 18 observers showed similar errors. The study by Becker (1935) shows that serial perception of two-dimensional shapes often leads to errors characterised by a failure to detect geometrical sub-components.

The failure to detect geometrical sub-components, associated with serial perception, has also been observed in a patient study by Behrmann, Winocur, and Moscovitch (1992). They found that a patient with severely impaired object identification (visual object agnosia) reproduced a configuration of two squares connected by a circle in a similar manner as observed by Becker (1935): instead of sketching the sub-components separately, the patient sketched the outer line connecting the three shapes. The finding of Behrmann et al. (1992) strengthens the hypothesis that serial perception causes impaired object identification. Our study has shown that the internal serial description of the stimulus can be used to sketch a recognisable drawing. Through sketching, it is possible to transform the difficult-to-identify serial description into an easier-to-identify simultaneous description. Therefore, we suggest that the difficulty of haptic line drawing perception is not caused by privileged object identification access of the visual system, but is caused by the inherent serial perception of the haptic system. The identification system is not well equipped to match the serial input to internal representations. There are possible ways to increase the haptic perceptual field, for instance, by using two hands, but haptic line drawings will still not be perceived as accurate and fast as visual line drawings.

\section{Acknowledgement}

This research was supported by a grant from the Netherlands Organisation for Scientific Research (NWO)

\section{References}

Becker, J. (1935). Über taktilmotorische Figurwahrnehmung. Psychological Research, 20(1), 102-158. 
Behrmann, M., Winocur, G., \& Moscovitch, M. (1992). Dissociation between mental imagery and object recognition in a brain damaged patient. Nature, 359, 636-637.

Chambers, D., \& Reisberg, D. (1985). Can mental images be ambiguous? Journal of Experimental Psychology: Human Perception and Performance, 11(3), 317-328.

D’Angiulli, A., Kennedy, J. M., \& Heller, M. A. (1998). Blind children recognizing tactile pictures respond like sighted children given guidance in exploration. Scandinavian Journal of Psychology, 39(3), $187-190$.

Finke, R. A., Pinker, S., \& Farah, M. J. (1989). Reinterpreting visual patterns in mental imagery. Cognitive Science, 13(1), 51-78.

Hayward, V. (in press). A brief taxonomy of tactile illusions and demonstrations that can be done in a hardware store. Brain Research Bulletin.

Heller, M. A. (1989). Picture and pattern perception in the sighted and the blind the advantage of the late blind. Perception, 18(3), 379-389.

Heller, M. A. (2002). Tactile picture perception in sighted and blind people. Behavioural Brain Research, 135(12), 65-68.

Heller, M. A., Calcaterra, J. A., Burson, L. L., \& Tyler, L. A. (1996). Tactual picture identification by blind and sighted people: Effects of providing categorical information. Perception \& Psychophysics, 58(2), 310-323.

Ikeda, M., \& Uchikawa, K. (1978). Integrating time for visual pattern perception and a comparison with the tactile mode. Vision Research, 18(11), 1565-1571.

Kennedy, J. M. (1993). Drawing and the blind. Yale University Press.

Kennedy, J. M., \& Bai, J. (2002). Haptic pictures: Fit judgments predict identification, recognition memory, and confidence. Perception, 31(8), 1013-1026.

Kennedy, J. M., \& Juricevic, I. (2006). Foreshortening, convergence and drawings from a blind adult. Perception, 35(6), 847-851.
Klatzky, R. L., Loomis, J. M., Lederman, S. J., Wake, H., \& Fujita, N. (1993). Haptic identification of objects and their depictions. Perception \& Psychophysics, 54(2), 170-178.

Kosslyn, S. (1994). Image and brain. The MIT Press.

Lederman, S. J., Klatzky, R. L., Chataway, C., \& Summers, C. D. (1990). Visual mediation and the haptic identification of 2-dimensional pictures of common objects. Perception \& Psychophysics, 47(1), 54-64.

Loomis, J. M., Klatzky, R. L., \& Lederman, S. J. (1991). Similarity of tactual and visual picture recognition with limited field of view. Perception, 20(2), 167-177.

Magee, L., \& Kennedy, J. (1980). Exploring pictures tactually. Nature, 283, 287-288.

Panis, S., De Winter, J., Vandekerckhove, J., \& Wagemans, J. (2008). Identification of everyday objects on the basis of fragmented outline versions. Perception, 37(2), 271-289.

Pylyshyn, Z. W. (2003). Seeing and visualizing: It's not what you think. The MIT Press.

Snodgrass, J., \& Vanderwart, M. (1980). A standardized set of 260 pictures: Norms for name agreement, image agreement, familiarity, and visual complexity. Journal of Experimental Psychology: Human Learning and Memory, 6, 174-215.

Thompson, L. J., Chronicle, E. P., \& Collins, A. F. (2003). The role of pictorial convention in haptic picture perception. Perception, 32(7), 887-893.

Verstijnen, I., Van Leeuwen, C., Goldschmidt, G., Hamel, R., \& Hennessey, J. (1998). Creative discovery in imagery and perception: Combining is relatively easy, restructuring takes a sketch. Acta Psychologica, 99, 177-200.

Wijntjes, M.W.A., Lienen, T. van, Verstijnen, I.M., \& Kappers, A.M.L. (in press). The influence of picture size on recognition and exploratory behaviour in raised line drawing perception. Perception, doi:10.1068/ p5714. 